\title{
Aggregation of polylactide with carboxyl groups at one chain end in the presence of metal cations
}

\author{
Melania Bednarek • Malgorzata Basko • \\ Tadeusz Biedroń • Przemysław Kubisa • \\ Mirosław Pluta
}

Received: 21 October 2013/Revised: 31 March 2014/Accepted: 8 April 2014/

Published online: 22 April 2014

(C) The Author(s) 2014. This article is published with open access at Springerlink.com

\begin{abstract}
Polylactides with one or more carboxyl groups at one chain end were synthesized by cationic polymerization according to activated monomer mechanism and by application of "thiol-yne" click chemistry for subsequent functionalization. End groups of such obtained polylactides were converted into ionic groups by neutralization of polymer solutions with metal oxides, mainly calcium oxide, and the aggregation of individual stereoisomers as well as that of the mixture of poly(Llactide) (PLLA) and poly(D-lactide) (PDLA) was investigated. The extent and progress of the aggregation was followed by viscosity measurements, and aggregated polymers in the solid state were examined by SEM and DSC. Solution viscosity increase was observed upon the aggregation of individual PLA stereoisomers, whereas PLA stereocomplex precipitation occurred in the case of the aggregation of PLLA/PDLA/metal oxide mixture.
\end{abstract}

Keywords Cationic polymerization - Biocompatible polymers $\cdot$ Polylactide . Aggregation

\section{Introduction}

Polylactide (PLA) as a biodegradable, biocompatible polyester has become one of the most intensively studied polymers in the recent years. Its existing and potential applications inspire many research groups to elaborate new methods of polymer synthesis or to improve those already existing, as well as to study the PLA's properties. With respect to biomedical applications, the behavior of polylactide in biological environment may be very important. Many aspects of

M. Bednarek $(\bowtie) \cdot$ M. Basko $\cdot$ T. Biedroń $\cdot$ P. Kubisa $\cdot$ M. Pluta

Center of Molecular and Macromolecular Studies, Polish Academy of Sciences, Sienkiewicza 112,

90-362 Lodz, Poland

e-mail: bednarek@cbmm.lodz.pl 
polymer interactions with organic or inorganic components of biological systems can be considered. Among them, the interaction of PLA chains with metal cations, e.g., $\mathrm{Ca}^{2+}$ naturally occurring in biological environment, is worth studying.

PLA synthesized by typically applied methods, i.e., by ring-opening polymerization of cyclic diester-lactide, usually contains carboxyl group at one chain end [1, $2]$. We have recently studied the aggregation of other biodegradable polyesterpoly( $\varepsilon$-caprolactone) (PCL), containing different numbers of carboxyl groups at one chain end, in the presence of calcium cations [3], and found that the extent of aggregation depended strongly on the number of carboxyl groups (strong synergistic effect) and that PCL chains containing calcium carboxylate groups formed long cylindrical aggregates.

Although the aggregation phenomena of polymers containing ionic groups along their polymer chain have been studied extensively [4, 5], only few papers appeared concerning the aggregation of polymers with an ionic group at one chain end [610]. Biocompatible polyesters have not been studied in this respect and our previous article concerning PCL aggregation was the first example. Consequently, we decided to extend our studies to another biocompatible polyester, namely polylactide containing carboxyl groups at its chain end, in the presence of metal cations.

Lactide, in contrast to previously studied caprolactone, contains a chiral center, thus it may exist in two enantiomeric forms. Polymerizations of L- or Dlactide lead to two enantiomeric forms of polymer, namely poly(L-lactide) or poly(D-lactide). It is known that poly(L-lactide) and poly(D-lactide) form stereocomplexes with physical properties different from those of individual stereoisomers [11]. Thus, aggregation of a mixture of enantiomeric polylactides containing ionic groups should involve, in addition to interaction of ionic groups observed earlier for polycaprolactone, also an interaction of two chains of opposite chirality.

The understanding of this combined effect may be interesting from scientific point of view as well as with respect to biomedical applications.

In this article, we describe the results of aggregation studies of PLA containing carboxyl groups at one chain end with consideration of both PLA stereoisomers. We also present methods of the synthesis of polylactide containing one or more carboxyl groups at one chain end. These methods are based on cationic polymerization of cyclic diester-lactide and on post-functionalization by applying "click chemistry" widely used in recent years in the synthesis of functional polymers [12-15]. Cationic polymerization performed in the presence of an initiator containing hydroxyl groups and additional functional group proceeds by activated monomer (AM) mechanism [16] and is a very convenient method of the synthesis of functional polymers.

Two different strategies were applied. The first was one-step synthesis by applying hydroxyacid as an initiator, while the second was two-step strategysynthesis of PLA with an unsaturated end group with subsequent functionalization by addition of a thiol containing carboxyl groups. 


\section{Experimental}

\section{Materials}

L-Lactide (L-LA, Boehringer Ingelheim, Germany) and D-lactide (D-LA, Purac) were crystallized from dry 2-propanol.

Glycolic acid was dried on vacuum line. Propargyl alcohol (99 \%, Aldrich) was stored over molecular sieves and distilled in vacuum before use.

Trifluoromethanesulfonic acid (triflic acid, $98 \%$, Aldrich), mercaptosuccinic acid (MSA, $99 \%$, Aldrich), 2,2-dimethoxy-2-phenylacetophenone (DMPA, $99 \%$, Aldrich), $\mathrm{CaO}$ (p.p.a., POCh, Poland), $\mathrm{Fe}_{2} \mathrm{O}_{3}$ (99\%, Aldrich), $\mathrm{ZnO}$ (99 \%, Aldrich) and cholecalciferol (vitamin $\mathrm{D}_{3}, 98 \%$, Sigma) were used as received.

Dichloromethane (p.p.a., POCh, Poland) and 1,4-dioxane (pure, Chempur, Poland) were dried over calcium hydride and distilled. 1,3-Dichloropropane (99\%, Aldrich), tetrahydrofuran (HPLC grade, Baker) diethyl ether (p.p.a., Chempur, Poland) and acetonitrile (HPLC grade, POCh, Poland) were used as received.

Synthesis of PLA with one carboxyl group at the chain end

A Schlenk tube was charged with L- or D-lactide $(2 \mathrm{~g}, 13.9 \mathrm{mmol})$ and glycolic acid ( $9.5 \mathrm{mg}, 0.125 \mathrm{mmol}$ ), closed with a rubber septum, degassed on the vacuum line and filled with nitrogen. The tube was placed in an oil bath and heated to $105^{\circ} \mathrm{C}$ to melt the monomer. Then triflic acid $(30 \mu \mathrm{L}$ of $10 \%$ solution in dichloroethane) was added with a syringe through the rubber septum. The polymerization was conducted at $\sim 105{ }^{\circ} \mathrm{C}$ in nitrogen atmosphere with stirring (magnetic stirrer) for about $3 \mathrm{~h}$ (after about $2.5 \mathrm{~h}$ solidification of polymer was observed, the polymerization vessel was heated for the next $0.5 \mathrm{~h}$ ). Solid polymer was crushed, washed three times with distilled water to remove catalyst and dried for $5 \mathrm{~h}$ at about $40{ }^{\circ} \mathrm{C}$ in vacuum. The polymer was analyzed by ${ }^{1} \mathrm{H}$ NMR and MALDI TOF.

Synthesis of PLA with propargyl group at the chain end

L- or D-Lactide (2 g, $13.9 \mathrm{mmol}$ ) was placed in a Schlenk tube which was degassed on vacuum line. The tube was filled with nitrogen, next dichloromethane was added with a syringe through the rubber septum followed by the addition of propargyl alcohol $(17 \mu \mathrm{L}, 16.5 \mathrm{mg}, 0.29 \mathrm{mmol})$ and triflic acid $(8 \mu \mathrm{L})$. Polymerization was conducted during about $18 \mathrm{~h}$ at room temperature with stirring (magnetic stirrer), then solvent was evaporated, solid polymer was crushed and washed three times with water. The polymer was analyzed by ${ }^{1} \mathrm{H}$ NMR and MALDI TOF. Mn $=\left({ }^{1} \mathrm{H}\right.$ NMR) $\sim 3,600$.

Coupling reaction of propargyl-PLA with mercaptosuccinic acid

$0.4 \mathrm{~g}$ of propargyl-PLA ( $\mathrm{Mn} \sim 3,600, \sim 0.1 \mathrm{mmol}$ of alkyne groups) together with $0.60 \mathrm{~g}$ of mercaptosuccinic acid $(4 \mathrm{mmol})$ and $\sim 3 \mathrm{mg}$ of DMPA $(\sim 0.012 \mathrm{mmol})$ were dissolved in $10 \mathrm{~mL}$ of THF in a round-bottom flask which was closed with a 
rubber septum. The flask was degassed on vacuum line (using a needle) and filled with nitrogen. This cycle was repeated three times. Next the reaction mixture was irradiated with UV lamp (365 $\mathrm{nm}$ ) for $110 \mathrm{~min}$ at room temperature with stirring. Then polymer was precipitated into cold diethyl ether and the precipitate (after decantation of the solvent) was washed twice with $\mathrm{Et}_{2} \mathrm{O}$. The polymer was dried on vacuum line.

Viscosity measurements of PLA- $(\mathrm{COOH})_{x}$ solutions after addition of $\mathrm{CaO}$

To compare the behavior of solutions of polymers with different numbers of $-\mathrm{COOH}$ groups in the presence of calcium cations, $10 \mathrm{wt} \%$ solutions of PL-LA$(\mathrm{COOH})_{1}$ and PL-LA- $(\mathrm{COOH})_{2.8}$ in 1,2-dichloropropane were prepared and twofold excess of $\mathrm{CaO}$ with respect to $-\mathrm{COOH}$ groups was added. Thus, $0.1 \mathrm{~g}$ of PLLA$(\mathrm{COOH})_{1}(\sim 0.027 \mathrm{mmol}$ of $-\mathrm{COOH}$ groups $)$ was dissolved in $1 \mathrm{~mL}$ of $1,2-$ dichloropropane and was mixed with $0.003 \mathrm{~g}$ of $\mathrm{CaO}(0.054 \mathrm{mmol})$. The suspension was vigorously stirred (magnetic stirrer) for about $15 \mathrm{~min}$ until the solution became almost clear. Then $\sim 0.3 \mathrm{~mL}$ of solution was transferred to the measuring cell of viscosimeter with a pipette, the cell was placed in the instrument and the viscosity was measured periodically without removing the solution from the measuring cell.

Preparation of PLA stereocomplex microparticles and encapsulation of vitamin $\mathrm{D}_{3}$

10 wt $\%$ solutions of PLLA- $(\mathrm{COOH})_{1}$ and PDLA- $(\mathrm{COOH})_{1}$ were prepared by dissolving $0.05 \mathrm{~g}$ of each polymer ( $\sim 0.013 \mathrm{mmol}$ of $-\mathrm{COOH}$ groups $)$ in $0.5 \mathrm{~mL}$ of 1,2-dichloropropane. Solutions were mixed together with $0.003 \mathrm{~g}$ of $\mathrm{CaO}$ ( $0.054 \mathrm{mmol}$, twofold molar excess with respect to the total number of $-\mathrm{COOH}$ groups). The suspension was vigorously stirred (magnetic stirrer) for about $15 \mathrm{~min}$ and then was left for about $2 \mathrm{~h}$. After that time two phases were well separated-the precipitate (fraction 1) at the bottom of the flask and the solution above (fraction 2). After decantation, the precipitate was dried in vacuum and analyzed by SEM.

In the attempt of vitamin $\mathrm{D}_{3}$ encapsulation during PLA stereocomplexation, the same procedure was applied, vitamin $\mathrm{D}_{3}(0.0157 \mathrm{~g}, 16 \mathrm{wt} \%$ with respect to PLAs) was added together with $\mathrm{CaO}$ during mixing of PLLA and PDLA solutions. Fraction 1 (precipitate) (0.063 g) and Fraction 2 (solution) (0.096 g) were separated. Both fractions were analyzed by ${ }^{1} \mathrm{H}$ NMR. Fraction 2 contained vitamin $\mathrm{D}_{3}$ with traces of PLA not involved in stereocomplex. Fraction 1 contained PLA and vitamin $\mathrm{D}_{3}\left({ }^{1} \mathrm{H}\right.$ NMR spectrum of precipitate and SEM picture are presented in Fig. 13).

Methods of materials analysis

${ }^{1} \mathrm{H}$ NMR spectra were recorded in $\mathrm{CDCl}_{3}$ using a Bruker DRX500 instrument operating at $500 \mathrm{MHz}$.

Size-exclusion chromatography (SEC) was performed using an Agilent Pump 1100 Series with Agilent G1379A Degasser and a set of two PL-Gel $5 \mu$ mixed-C 
columns. Wyatt Optilab Rex interferometric refractometer and multi-angle laser light scattering (MALLS) Dawn Eos laser photometer (Wyatt Technology Corp., Santa Barbara, CA) were used as detectors. Dichloromethane was used as an eluent at a flow rate of $0.8 \mathrm{~mL} \mathrm{~min} \mathrm{~m}^{-1}$ at room temperature. The system was calibrated according to polystyrene standards.

Matrix-assisted laser desorption/ionization time-of-flight (MALDI TOF) measurements were performed with the Voyager Elite (PerSeptive Biosystems, Framingham, MA) time-of-flight instrument equipped with a pulsed $\mathrm{N}_{2}$ laser $(337 \mathrm{~nm})$ and time-delayed extraction source. The accelerating voltage of $20 \mathrm{kV}$ was used. Dithranol was used as a matrix, $\mathrm{CF}_{3} \mathrm{COOK}$ as a cationating agent and THF as a solvent.

Viscosity measurements were performed at $25^{\circ} \mathrm{C}$ using Brookfield DV$\mathrm{II}+$ viscosimeter with $\mathrm{S} 52$ spindle and shear rate (SR) of $10 \mathrm{~s}^{-1}$ (which corresponds to 5 RPM spindle rotation).

Scanning electron microscopy (SEM) images were taken using Jeol JSM$5500 \mathrm{LV}$ apparatus working in the secondary electron mode with an accelerating voltage of $10 \mathrm{kV}$.

Differential scanning calorimetry (DSC) analysis was performed under nitrogen at a heating and cooling rate of $10{ }^{\circ} \mathrm{C} / \mathrm{min}$ on DSC 2920 Modulated TA Instrument. Both temperature and heat flow were calibrated with indium.

Wide angle X-ray scattering (WAXS) analysis was performed by measuring X-ray intensity versus $2 \theta$ (from $10^{\circ}$ to $40^{\circ}$ ) in the transmission mode-coupled $\theta / 2 \theta$. A wide-angle computer controlled goniometer coupled to a sealed-tube source of filtered $\mathrm{Cu} \mathrm{K} \alpha$ radiation operating at $30 \mathrm{kV}$ and $50 \mathrm{~mA}$ (Philips PW3830) was used. The split system of the diffractometer was adjusted to measure the integral intensity of a given diffraction peak.

\section{Results and discussion}

Synthesis of PLA with one carboxyl group at the chain end

Cationic polymerization in which hydroxyacids were used as initiators was successfully used by us earlier for the synthesis of poly( $\varepsilon$-caprolactone) containing different numbers of $-\mathrm{COOH}$ groups at one chain end [3]. We tried to apply the same method to the synthesis of functional PLAs. Thus, poly(L-lactide) (PLLA) and poly(D-lactide) (PDLA) with one carboxyl group at the chain end were obtained by cationic bulk polymerization performed in the presence of glycolic acid as an initiator as it is shown in Fig. 1.

Cationic polymerization, in contrast to anionic or coordination polymerization, proceeds smoothly in the presence of free carboxyl groups. Bulk polymerization of lactide was performed at $105{ }^{\circ} \mathrm{C}$, i.e., slightly above the monomer melting point $\left(T_{\mathrm{m}}\right.$ of lactide $\left.=96^{\circ} \mathrm{C}[17]\right)$.

In GPC chromatograms monomodal molecular weight distribution was observed but the determination of true $M_{n}$ values from GPC analysis is doubtful because of the lack of appropriate standards (in some publications correction factors are used 


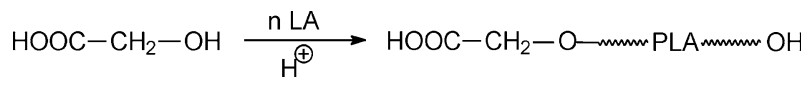

Fig. 1 Schematic representation of the synthesis of PLA with one carboxyl group at one chain end

but such procedure may be questioned for relatively low molecular weight polymers) $[18,19]$. Therefore, for the determination of $M_{n}$ we relied mainly on ${ }^{1} \mathrm{H}$ NMR analysis which allowed the calculation of molecular weights more reliably by comparison of the intensities of signals corresponding to terminal methine group with those corresponding to methine groups from polymer backbone. On the basis of ${ }^{1} \mathrm{H}$ NMR spectra number average molecular weights $M_{n}$ were determined as equal to $\sim 3,600$ for both PLLA-COOH and for PDLA-COOH.

MALDI TOF analysis (see Fig. 2) showed, however, that only part of macromolecules was initiated with glycolic acid and about half of the whole macromolecules population was initiated with water. Water was probably formed at elevated temperature as a by-product of condensation of hydroxyacid or its higher analogs. Fortunately macromolecules initiated with water have also $-\mathrm{COOH}$ and $-\mathrm{OH}$ end groups, thus, from the point of view of end-group structure, both series of macromolecules are almost the same, which is important for our studies.

Synthesis of PLA with several carboxyl groups at one chain end

First approach to the synthesis of polylactide with several carboxyl groups at one chain end was based on the same strategy as the synthesis of PLA with one - $\mathrm{COOH}$ group. Thus, cationic polymerization was performed in the presence of hydroxy acids with two (malic acid) or three (citric acid) carboxyl groups. Such method was successfully used earlier for polymerization of $\varepsilon$-caprolactone [3]. First experiments

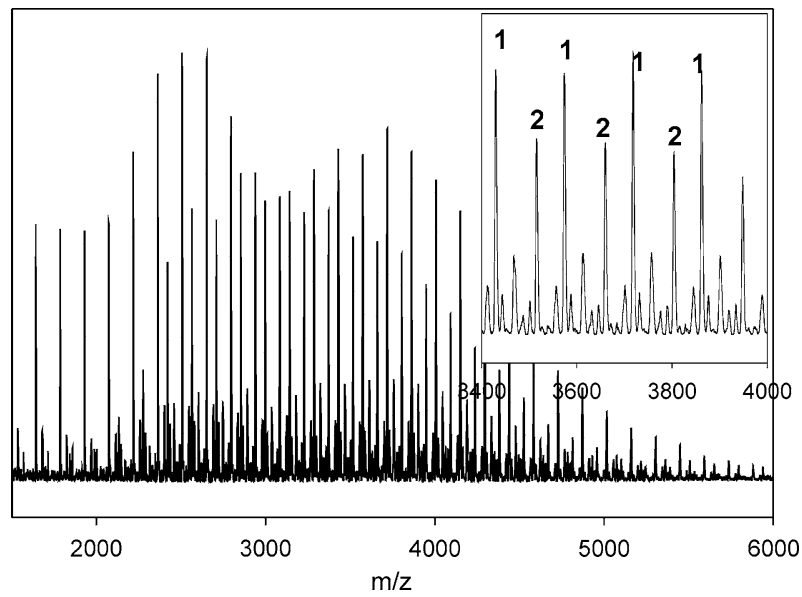

Fig. 2 MALDI TOF spectrum of PDLA-COOH obtained by polymerization initiated with glycolic acid: series 1 corresponds to macromolecules initiated with glycolic acid, series 2 corresponds to macromolecules initiated with water 
concerning bulk or solution cationic lactide polymerization showed, however, that it is not possible to obtain fully functionalized polymers by this approach. MALDI TOF analysis of obtained products indicated that significant fraction (over $80 \%$ ) of macromolecules was initiated with water. Water was present in the polymerization system as a by-product of the condensation reaction or dehydration product of $\beta$ hydroxyacids. Dehydration is competitive to polymerization and was fast enough in comparison with the initiation of polymerization by hydroxyacid to generate a new much more reactive initiating molecule, i.e., water (dehydration was not competitive in the case of the much more reactive monomer- $-\varepsilon$-caprolactone). The initiation of polymerization with water resulted in macromolecules containing one $-\mathrm{COOH}$ group at the chain end so this method could not lead to PLAs with two or three $-\mathrm{COOH}$ groups at the chain end. In this situation another strategy was applied. The PLA functionalization was achieved in two steps. First step was the synthesis of PLA with unsaturated end group to which, in the next step, a thiol containing $-\mathrm{COOH}$ groups was attached. We have recently described the results of such functionalization and compared several combinations of PLAs, thiols containing $-\mathrm{COOH}$ groups and reaction conditions [20]. The application of thiol-yne coupling by which two thiol molecules could be attached to one PLA chain seemed to be most promising. Thus, functionalized PLA with more than one carboxyl group at the chain end was obtained by the synthesis of PLA with propargyl end group with subsequent thiol-yne coupling with mercaptosuccinic acid. Figure 3 presents the applied strategy.

Efficiencies of both stages were checked by ${ }^{1} \mathrm{H}$ NMR and MALDI TOF analysis. As it was found, in the first step PLA with almost all (over $95 \%$ ) macromolecules functionalized with alkyne end groups was obtained. The efficiency of functionalization by thiol-yne coupling was not so good (see Fig. 4) which was in accordance with our earlier observation [20].

In the MALDI spectrum of the product of coupling reaction (Fig. 4) four main series of signals are visible. The assignment of signals is complicated because of the overlapping of signals of macromolecules with different structures. As it can be seen in Fig. 4a, all signals in the groups 1 and 2 are wide, as they contain several signals. Signals $1 \mathrm{~A}$ and $2 \mathrm{~A}$ are narrower. The MALDI spectrum shown in Fig. $4 \mathrm{~b}$ registered in reflector mode displays a splitting of main signals which confirms the presence of different signals under the wide signal. Thus, we concluded that Signal

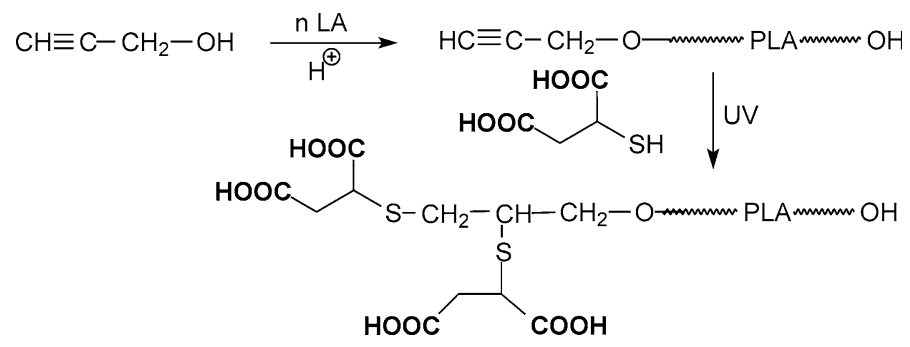

Fig. 3 Schematic representation of the synthesis of PLA with several carboxyl groups at one chain end 


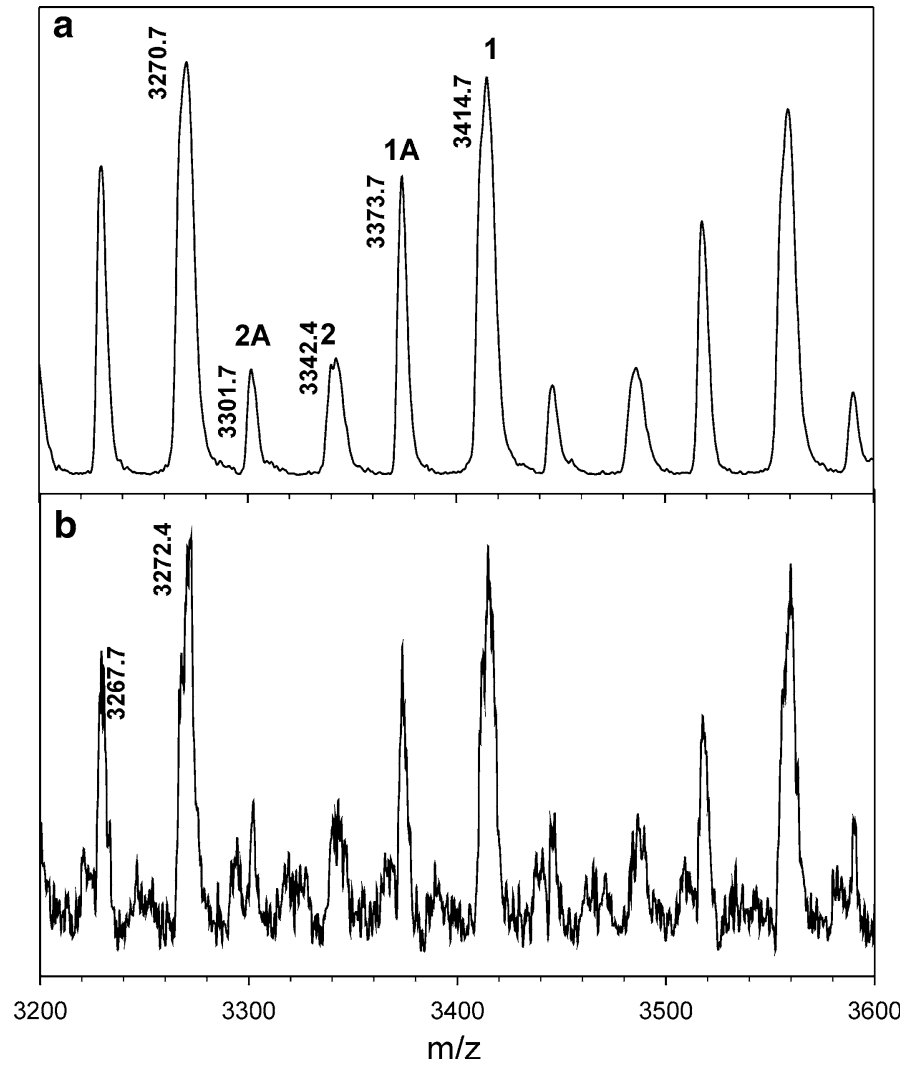

Fig. 4 Fragments of MALDI TOF spectra of the product of coupling reaction of propargyl-PDLA with MSA: a registered in linear mode, $\mathbf{b}$ registered in reflector mode

1 around $\mathrm{m} / \mathrm{z} \sim 3,414$ corresponds to functionalized macromolecules: $M_{1}=21 \times$ $144.13+56+150+150+39=3,421.7$ and $M_{2}=22 \times 144.13+56+$ $150+39=3,415.9$ and unfunctionalized macromolecule: $M_{3}=23 \times 144.13+$ $56+39=3,410.0$. Signal $1 \mathrm{~A}$ around $\mathrm{m} / \mathrm{z} \sim 3,373$ corresponds to macromolecules cationated with proton (instead of potassium): $M_{4}=22 \times 144.13+56+$ $150+1=3,377.9$ and $M_{5}=23 \times 144.13+56+1=3,372.0$. Signal 2 around $m / z \sim 3,342$ corresponds to macromolecules which underwent transesterification: $M_{6}=41 \times 72.07+56+150+150+39=3,349.9$ and $M_{7}=43 \times 72.07+$ $56+150+39=3,344.0$ and possibly also unfunctionalized macromolecules: $M_{8}=45 \times 72.07+56+39=3,338.2$. Signal $2 \mathrm{~A}$ around $m / z \sim 3,301$ corresponds to transesterified macromolecules cationated with proton: $M_{9}=43 \times$ $72.07+56+150+1=3,306.0$ and unfunctionalized ones: $M_{10}=45 \times$ $72.07+56+1=3,300.2$.

Summarizing the analysis of MALDI TOF spectra recorded for the product of thiol-yne coupling between propargyl-PDLA and MSA (mercaptosuccinic acid), the majority of macromolecules were functionalized with two thiol molecules but 
unfunctionalized ones and those functionalized with one thiol molecule were also present.

Thus, MALDI TOF analysis cannot give unequivocal information about the average functionalization efficiency. In this situation, we rather relied on ${ }^{1} \mathrm{H}$ NMR analysis. On the basis of ${ }^{1} \mathrm{H}$ NMR spectrum presented in Fig. 5 the functionalization degree of propargyl-PDLA with MSA was estimated as $\sim 70 \%$ (similar value was found in the case of propargyl-PLLA functionalization), with an assumption that two thiol molecules can be attached to one alkyne group. Calculations were performed by comparison of the intensity of methylene and methine groups in the vicinity of sulfur present in attached thiol group with the intensity of methine endgroup - $\mathrm{CH}\left(\mathrm{CH}_{3}\right)-\mathrm{OH}$ or by comparison of the intensity of methylene from residual unreacted propargyl group with the intensity of $-\underline{\mathrm{CH}}\left(\mathrm{CH}_{3}\right)-\mathrm{OH}$ group.

Thus, we concluded that although the applied method did not yield a uniform product of desired structure, majority of macromolecules indeed contained several carboxyl groups at the chain end. Such products were used for aggregation studies.

Aggregation of PLA with carboxyl groups at the chain end in the presence of metal cations

\section{Aggregation of PLLA-(COOH $)_{x}$ stereoisomer}

Results of earlier studies indicated that polymers containing ionic groups at the chain end tend to form spherical or cylindrical aggregates in solution in nonpolar solvent, in the presence of metal cations [3, 6, 9]. Shapes and sizes of aggregates depend on many factors. The results of our previous work [3] concerning the aggregation of poly( $\varepsilon$-caprolactone) polymers with different numbers of carboxyl groups at one chain end showed that the aggregation tendency depended on the concentration and molecular weight of polymer, the excess of metal cations with

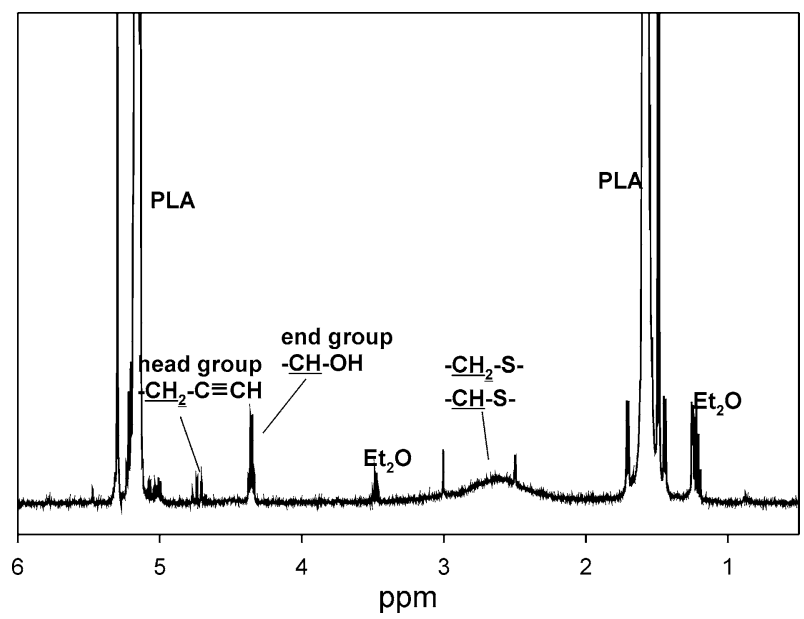

Fig. $5{ }^{1} \mathrm{H}$ NMR spectrum of the product of coupling reaction of propargyl-PDLA with MSA 


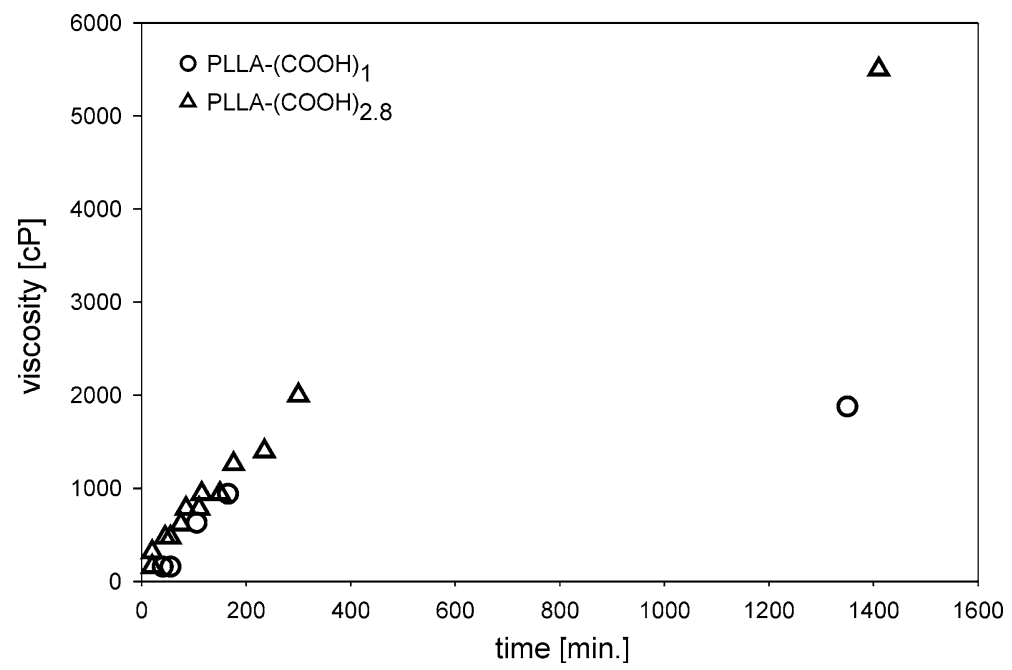

Fig. 6 The evolution of viscosity of 1,3-dichloropropane solutions of PLLA polymers after addition of $\mathrm{CaO} ;[\mathrm{CaO}] /[-\mathrm{COOH}]=2$

respect to $-\mathrm{COOH}$ groups, the size of metal cation, but most of all on the number of ionic groups. The aggregation progress, after addition of metal oxide, manifested itself in gradual increase of solution viscosity (usually from about $100 \mathrm{cP}$ to $\left.10^{4} \div 10^{6} \mathrm{cP}\right)$ up to the formation of stable polymer gels. SEM analysis of dried PCL- $(\mathrm{COOH})_{x}$ gels formed upon the aggregation indicated the formation of long cylindrical structures.

The aggregation tendency of PLA containing carboxyl groups at the chain end was investigated, similarly to PCL polymers [3], by viscosity measurements. Aggregations of two PLA samples were compared: poly(L-LA) with only one $-\mathrm{COOH}$ group at the chain end obtained by cationic polymerization initiated with glycolic acid and poly(L-LA) with more than two - $\mathrm{COOH}$ groups on average (PLLA- $\left.(\mathrm{COOH})_{2.8}\right)$ obtained by polymerization initiated by propargyl alcohol and coupled with mercaptosuccinic acid. Figure 6 presents viscosity-time profiles obtained as a result of viscosity measurements performed with 1,2-dichloropropane solutions of those PLLAs after addition of calcium oxide.

The solution viscosities increased, although these increases were not as significant as observed previously for PCL- $(\mathrm{COOH})_{x}$ polymers and gels which formed in the end were not stable (the gels destroyed upon shaking). In spite of low solution viscosity increases, higher value of viscosity was achieved in the case of PLA with a higher number of carboxyl groups.

Relatively modest increase of viscosity (much lower than in the case of the earlier studied $\varepsilon$-caprolactone polymers) indicated a low tendency to aggregation. That was confirmed by SEM analysis of products after solvent evaporation (Fig. 7).

As it can be seen in Fig. 7, no specific forms can be detected in the SEM images of dried PLLA gels after the aggregation. Rather moderate viscosity increases upon the neutralization of PLLAs solutions with calcium cations and the absence of 

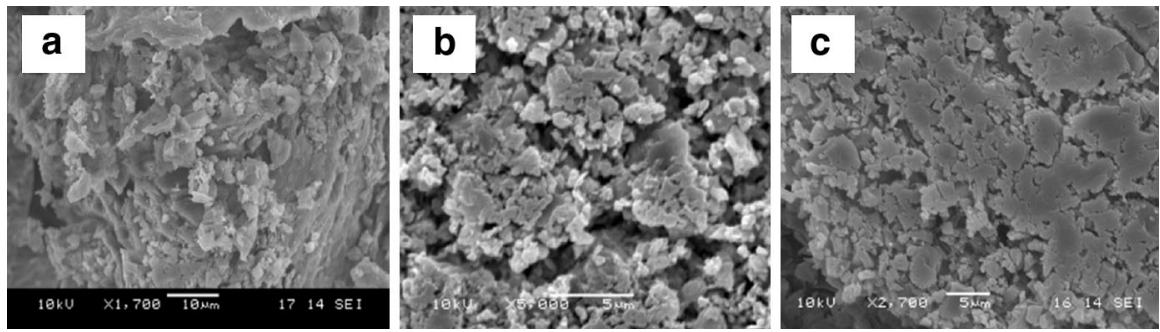

Fig. 7 SEM images of PLLA- $(\mathrm{COOH})_{1} / \mathrm{CaO}$ a and PLLA- $(\mathrm{COOH})_{2.8} / \mathrm{CaO}, \mathbf{b}$ after drying of gels obtained at the end of polymers aggregation in 1,3-dichloropropane solutions; The picture of PLLA$(\mathrm{COOH})_{1}$ before aggregation is also shown for comparison (c)

detectable forms in SEM pictures of materials obtained after the aggregation indicated a weak tendency of polylactide with carboxyl groups to the aggregation in a solvent of low polarity. It was interesting to find whether this tendency could change when the aggregation was performed in the system of both polylactide stereoisomers.

\section{Aggregation of PLLA-(COOH $)_{x}$ and PDLA- $(\mathrm{COOH})_{x}$ mixture}

The viscosity changes of mixed, separately prepared solutions of PLLA-COOH and PDLA-COOH after the addition of $\mathrm{CaO}$ were followed by Brookfield instrument. It appeared that in this case viscosity of the mixture almost did not change and polymer precipitation from the solution was observed instead. The formed precipitate was PLA stereocomplex. Figure 8 presents the SEM image of the precipitate obtained after the aggregation of both stereoisomers in the presence of calcium oxide.

In the presented SEM picture of the precipitate, among irregular small particles (similar to that of dried gels from the aggregation of one stereoisomer), also uniform microspheres with a diameter of about 1.5-2 $\mu \mathrm{m}$ are present. The phenomenon of microspheres formation was observed sporadically in the case of PLA stereocomplex synthesis from equimolar PLA stereoisomers solutions when specially chosen conditions were applied [21]. We have recently found that regular microspheres were formed in the case of stereocomplexation of PLA with ionic groups at the chain end [22]. Evidently in the system studied by us the addition of calcium oxide to PLA-COOH solutions caused ionization of carboxyl groups. Shapes and sizes of stereocomplex microparticles depend on conditions of their crystallization from a solution. In our study, we did not optimize precipitation conditions, however, we checked the influence of solvent polarity and metal cation on facilitating the ionization of $-\mathrm{COOH}$ groups. The appearance of microparticles obtained in chosen solvents using several metal oxides is shown in Fig. 9.

1,3-Dichloropropane seems to favor the formation of spherical particles. We, therefore, chose this solvent for the aggregation in the presence of oxides other than $\mathrm{CaO}$. Metal originated from the introduced oxide remained inside the particles, which could be useful in potential applications of PLA microparticles such as 


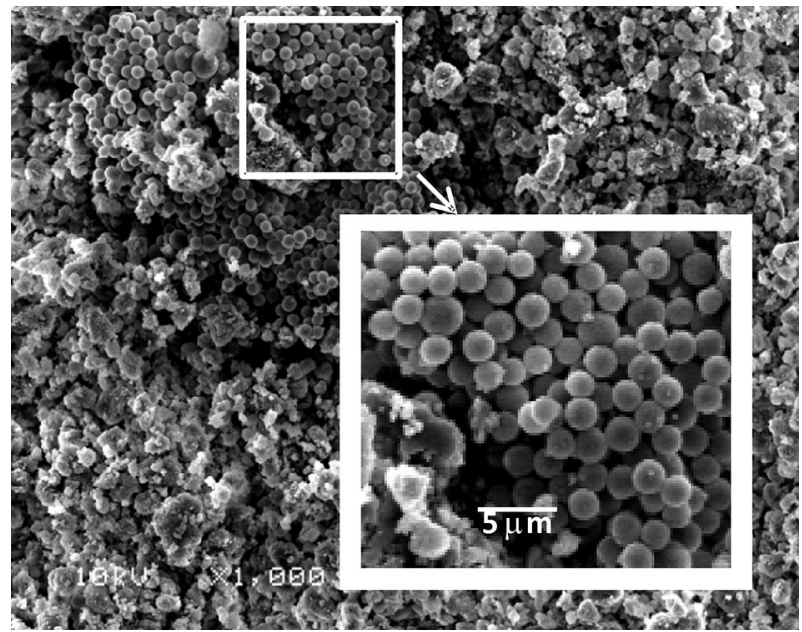

Fig. 8 SEM image of stereocomplex formed of PLLA-COOH, PDLA-COOH and $\mathrm{CaO}$; in the inlet an area in which regular microspheres are present is shown
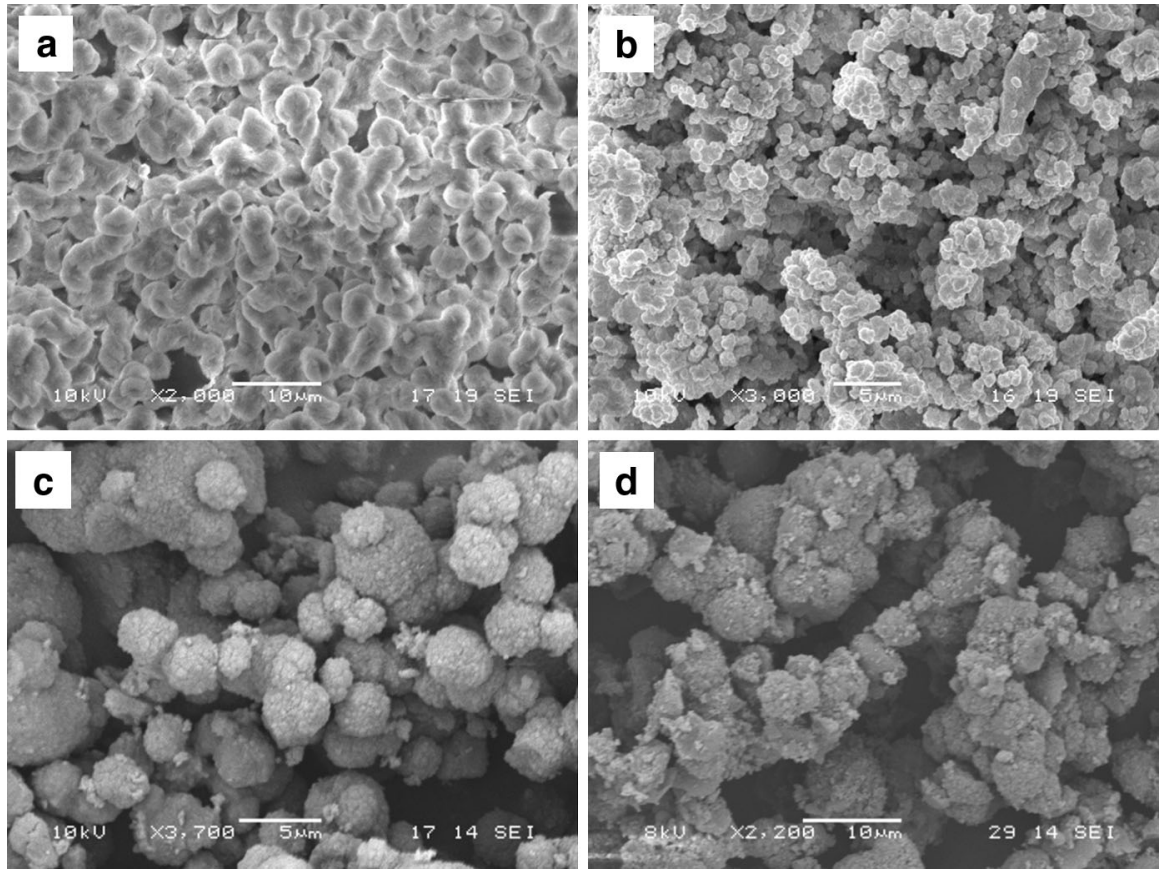

Fig. 9 SEM images of aggregation products of PLLA-COOH/PDLA-COOH performed in the presence of $\mathrm{CaO}$ in 1,4-dioxane $\mathbf{a}$ or in acetonitrile, $\mathbf{b}$ and performed in the presence of $\mathrm{Fe}_{2} \mathrm{O}_{3}$, $\mathbf{c}$ or in the presence of $\mathrm{ZnO}$, $\mathbf{d}$ in 1,3-dichloropropane 
biomedical PLA-based materials or microparticles with iron oxide which display magnetic properties. DSC analysis of the precipitate indeed confirmed that a stereocomplex was formed. In DSC thermograms presented in Fig. 10 melting peaks corresponding to the aggregation product obtained in the system with both PLA-COOH stereoisomers appeared at a significantly higher temperature than peaks corresponding to the aggregation product of PLLA-COOH and to the starting PLLA-COOH (although all melting temperatures are lower than those for high molecular PLA cited in the literature).

The stereocomplex formation upon aggregation of PLLA-COOH/PDLA-COOH/ $\mathrm{CaO}$ system was also evidenced by WAXS analysis. In WAXS profile (solid line in Fig. 11) the peaks characteristic for PLA stereocomplex [11] can be identified at $2 \theta$ equal to $12.0^{\circ}, 20.9^{\circ}$ and $24.1^{\circ}$. WAXS profile obtained for the aggregation product of only one PLA stereoisomer is also shown in Fig. 11 for comparison.

Aggregation with the participation of both stereoisomers was also performed for polylactide with more than one $-\mathrm{COOH}$ group at one chain end. Equimolar solutions of PLLA- $(\mathrm{COOH})_{2.8}$ and PDLA- $(\mathrm{COOH})_{2.8}$ in 1,3-dichloropropane were mixed together with adding $\mathrm{CaO}$. Figure 12 presents a SEM image of stereocomplex particles. In the figure, spherical particles of about $2 \mu \mathrm{m}$ diameter are visible. The lack of very regular microspheres could be explained by the presence of

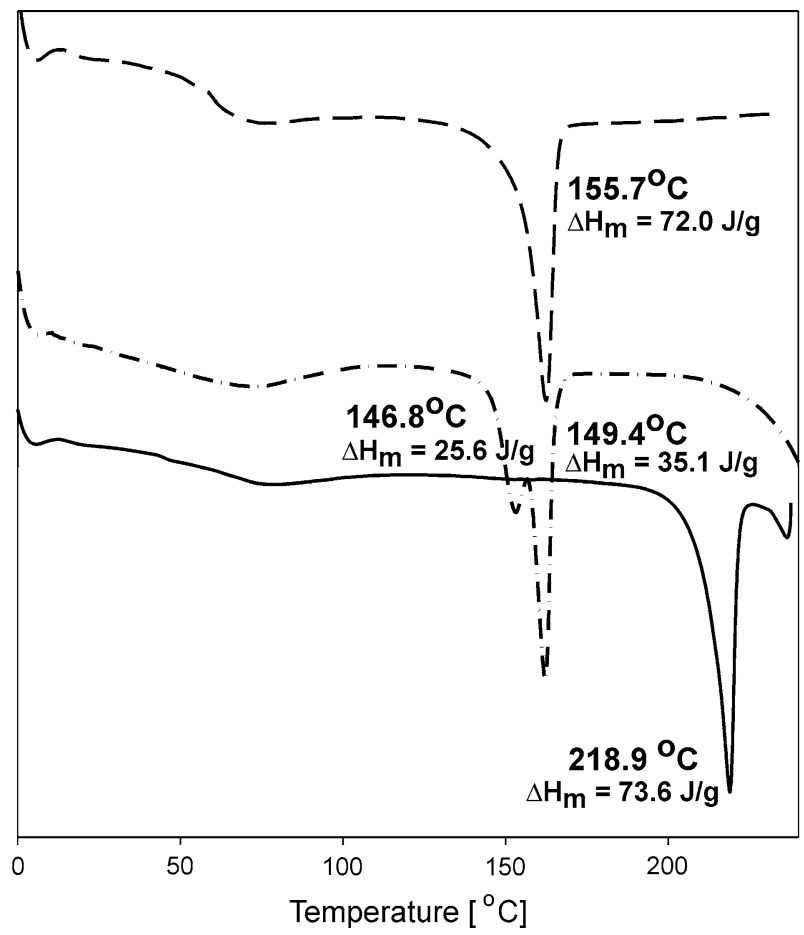

Fig. 10 DSC analysis of the precipitate obtained as result of aggregation in the system with both PLA$\mathrm{COOH}$ stereoisomers (solid line); for comparison thermograms of the aggregation product of PLLA$\mathrm{COOH}$ (dashed line) and of the starting PLLA-COOH (dashed-dotted line) are shown 


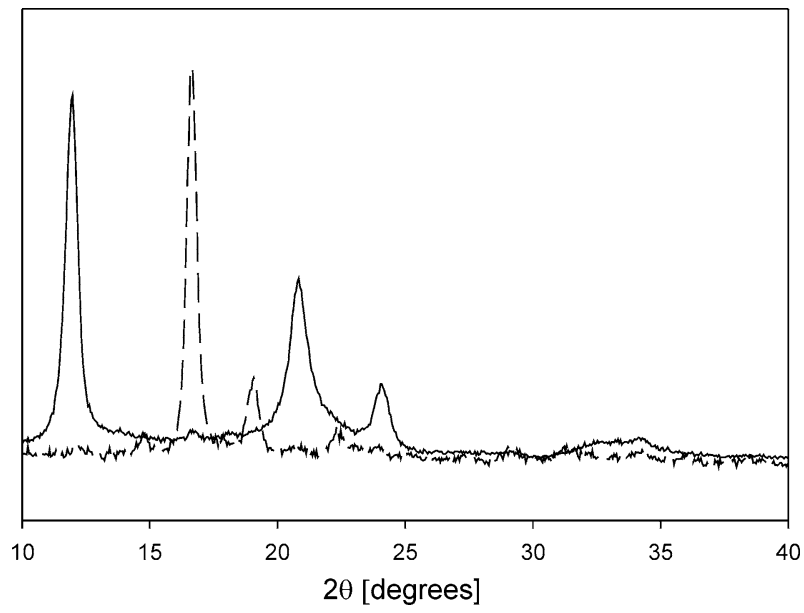

Fig. 11 WAXS profiles of PLLA-COOH/PDLA-COOH/CaO (solid line) and PLLA-COOH//CaO (dashed line) aggregation products

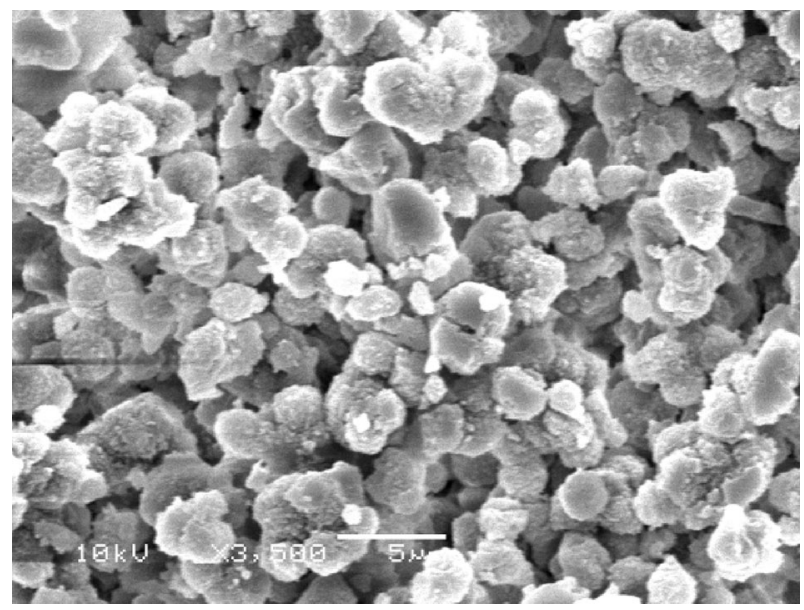

Fig. 12 SEM image of stereocomplex formed of PLLA- $(\mathrm{COOH})_{2.8}, \mathrm{PDLA}-(\mathrm{COOH})_{2.8}$ and $\mathrm{CaO}$

macromolecules with different functionalities and larger diversity of polymer end groups' interactions with metal cations in the case of PLA with attached thiol molecules.

Phenomena of the formation of small spherical PLA particles with the diameter of a few micrometers may find an application in the encapsulation of active compounds for biomedical purposes. Great efforts are undertaken to develop polymer carriers, including solid nano- and microparticles, for pharmaceutical agents to expand the utility of drugs for various clinical applications. To place a pharmaceutical agent inside the microparticle, the entrapping methods were 

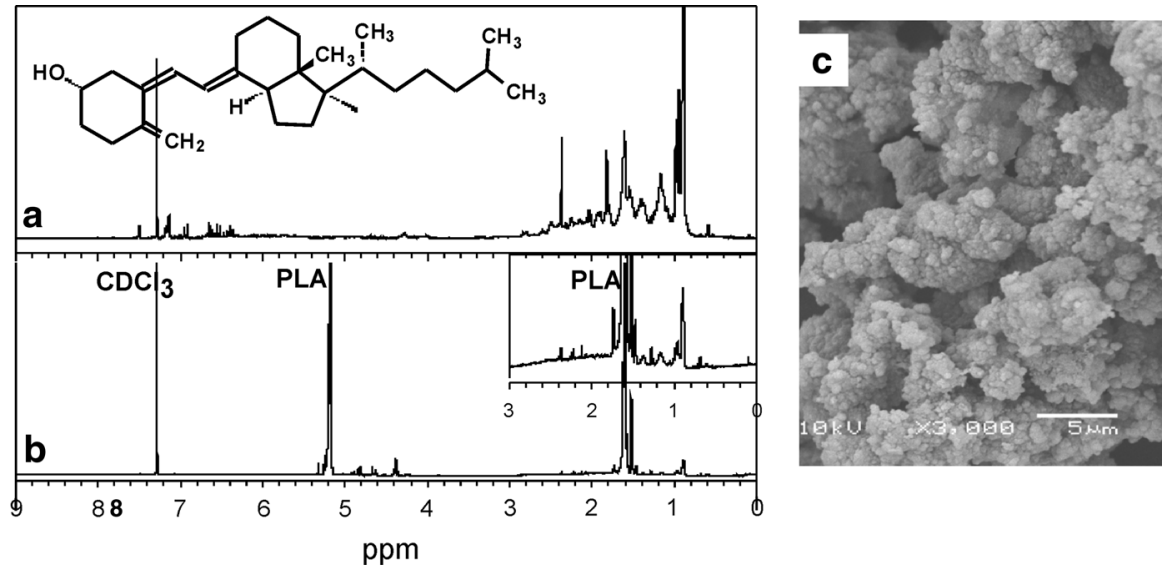

Fig. $13{ }^{1} \mathrm{H}$ NMR spectra of vitamin $\mathrm{D}_{3}$ (a), product of aggregation of the PLLA-COOH/PDLA-COOH/ $\mathrm{CaO} /$ vitamin $\mathrm{D}_{3}$ system (b) and SEM image of this product (c)

developed which are based mainly on emulsion techniques like water in oil in water $(\mathrm{w} / \mathrm{o} / \mathrm{w})$, water in oil in oil (w/o/o) and solid in oil in oil (s/o/o) emulsions [23]. Emulsion/solvent diffusion techniques have also been applied for drugs encapsulation into PLA (PLA copolymers) microspheres [24, 25]. We decided to take an advantage of spontaneous microparticles formation during PLA stereocomplex precipitation for the entrapment of pharmaceutical agents. When microspheres are formed during PLA stereocomplexation, the whole process of emulsification, and therefore the choice of emulsification conditions, can be avoided. Metal cations present in the system can act as a stabilizing agent [25, 26]. Additionally, the presence of metal cations could be advantageous for the pharmaceutical agent assimilation, regarding potential applications. Thus, vitamin $\mathrm{D}_{3}$ (cholecalciferol) is frequently administered together with $\mathrm{Ca}^{2+}$ [27]. First attempts of the PLA stereoisomers aggregation in the presence of several vitamins indicated a loading degree of several percent. Figure $13 \mathrm{a}, \mathrm{b}$ presents ${ }^{1} \mathrm{H}$ NMR spectra indicating the presence of vitamin $\mathrm{D}_{3}$ inside particles and Fig. 13c presents a SEM image of the formed microparticles loaded with the vitamin and $\mathrm{CaO}$.

Although the vitamin loading degree is not high (around $4 \mathrm{wt} \%$ ) it can be quite sufficient in the therapeutic application assuming that the typical supplementing dose per day is around $10 \mu \mathrm{g}$ applicable in $0.25 \mathrm{~g}$ of oil. The work concerning the encapsulation of different pharmaceutical agents during PLA stereocomplexation with a possible support of specific interactions between aggregating system components is in progress.

\section{Conclusions}

Poly(lactide) containing carboxyl groups at one chain end undergoes the aggregation in the presence of metal cations in nonpolar solvent. The aggregation 
phenomena proceed in different ways depending whether one PLA stereoisomer or a mixture of stereoisomers is used. In the case of one PLA- $(\mathrm{COOH})_{x}$ stereoisomer, polymer chains undergo self-organization by the interaction of terminal carboxyl groups with metal cations. Such aggregation leads to solution viscosity increase and formation of gels but no defined morphology of aggregates is observed in the dried polymer. The aggregation tendency depends on the number of carboxyl groups.

The aggregation of equimolar mixture of PLA stereoisomers-PLLA-COOH and PDLA-COOH-involves two phenomena, i.e., the interaction of terminal carboxyl groups with metal cations and the interaction between two complementary polylactide (PLLA and PDLA) chains. Such interactions lead to self-organization resulting in the formation of spherical microparticles which contain a metal ion inside. The spontaneous formation of microparticles may find an application in pharmaceutical agent's encapsulation as it was preliminarily shown for vitamin $\mathrm{D}_{3}$ / calcium combination.

Acknowledgment Financial support by the National Science Centre (Grant No N204 131940) is gratefully acknowledged.

Open Access This article is distributed under the terms of the Creative Commons Attribution License which permits any use, distribution, and reproduction in any medium, provided the original author(s) and the source are credited.

\section{References}

1. Duda A, Penczek S (2002) Mechanisms of aliphatic polyester formation. In: Steinbüchel A, Doi Y (eds) Biopolymers, polyesters II-properties and chemical synthesis, vol 3B. Wiley, Weinheim, pp 371-429

2. Gruber P, O’Brien M (2002) Polylactides "Nature Works ${ }^{\mathrm{TM}}$ PLA". In: Steinbűchel A, Doi Y (eds) Biopolymers, polyesters III-applications and commercial products, vol 4. Wiley, Weinheim, pp 235-250

3. Bednarek M, Biedroń T, Kubisa P (2010) Aggregation of $\varepsilon$-caprolactone polymers containing at one chain end different number of carboxyl groups in the presence of calcium cations. J Polym Sci Part A: Polym Chem 48:5630-5635. doi:10.1002/pola.24353

4. Eisenberg A (1970) Clustering of ions in organic polymers. A theoretical approach. Macromolecules 3:147-154. doi:10.1021/ma60014a006

5. Eisenberg A, King M (1977) In: Stein R (ed) Ion-containing polymers: physical properties and structure. Polymer Physics Series, vol 2. Academic Press, New York, pp 15-64

6. Wang Z-G (1990) Aggregation (micellization) of associating polymers. Langmuir 6:928-934. doi:10. 1021/la00095a007

7. Vanhoorne P, Jerome R (1995) Aggregation behavior of omega- and alpha, omega-metal sulfonato polystyrene in toluene. Macromolecules 28:5664-5670. doi:10.1021/ma00120a036

8. Zhong XF, Eisenberg A (1994) Aggregation and critical micellization behavior of carboxylateterminated monochelic polystyrene. Macromolecules 27:1751-1758. doi:10.1021/ma00085a013

9. Jalal N, Duplessix R (1988) Aggregation of monocarboxylic polymer chains by neutralization. Neutron and $X$ ray scattering. Journal de Physique 49:1775-1783. doi:10.1051/jphys: 0198800490100177500

10. Mucyn N, Duval M, Duplessix R (2001) Static and dynamic light scattering from monofunctional ionomer solutions. Aggregation of the $\omega$-carboxylic functionalized polystyrene through neutralization in tetrahydrofuran. J Macromol Sci Part B 40:1109-1130. doi:10.1081/MB-100107805 
11. Tsuji H (2005) Poly(lactide) Stereocomplexes: formation, structure, properties, degradation and applications. Macromol Biosci 5:569-597. doi:10.1002/mabi.200500062

12. Hawker CJ, Fokin VV, Finn MG, Sharpless KB (2007) Bringing efficiency to materials synthesis: the philosophy of click chemistry. Aust J Chem 60:381-383. doi:10.1071/CH07107

13. Hoogenboom R (2010) Thiol-yne chemistry: a powerful tool for creating highly functional materials. Angew Chem Int Ed 49:3415-3417. doi:10.1002/anie.201000401

14. Iha RK, Wooley KL, Nystrom AM, Burke DJ, Kade MJ, Hawker CJ (2009) Applications of orthogonal "Click" chemistries in the synthesis of functional soft materials. Chem Rev 109:5620-5686. doi:10.1021/cr900138t

15. Lowe AB (2010) Thiol-ene "click" reactions and recent applications in polymer and materials synthesis. Polym Chem 1:17-36. doi:10.1039/b9py00216b

16. Kubisa P, Penczek S (1999) Cationic activated monomer polymerization of heterocyclic monomers. Prog Polym Sci 24:1409-1437. doi:10.1016/S0079-6700(99)00028-3

17. Groot W, Van Krieken J, Sliekersl O, De Vos S (2010) Production and purification of lactic acid and lactide. In: Auras R, Lim L-T, Selke SEM, Tsuji H (eds) Poly(lactic acid): synthesis, structures, properties, processing, and applications., Polymer engineering and technology seriesWiley, New Jersey, pp 3-18

18. Van Dijk JAP, Smit JAM, Kohn FE, Feijen J (1983) Characterization of poly(D, L-lactic acid) by gel permeation chromatography. J Polym Sci, Part A: Polym Chem 21:197-208. doi:10.1002/pol.1983. 170210121

19. Kowalski A, Duda A, Penczek S (1998) Polymerization of L, L-lactide initiated by aluminum isopropoxide trimer or tetramer. Macromolecules 31:2114-2122. doi:10.1021/ma971737k

20. Bednarek M (2013) Coupling reaction with thiols as the efficient method of functionalization of "clickable" polylactide. React Funct Polym 73:1130-1136. doi:10.1016/j.reactfunctpolym.2013.04. 001

21. Tsuji H, Hyon S-H, Ikada Y (1992) Stereocomplex formation between enatiomeric poly(lactic acid)s. 5. Calorimetric and morphological studies on the stereocomplex formed in acetonitrile solution. Macromolecules 25:2940-2946. doi:10.1021/ma00037a024

22. Biedron T, Brzezinski M, Biela T, Kubisa P (2012) Microspheres from stereocomplexes of polylactides containing ionic liquid end-groups. J Polym Sci Part A: Polym Chem 50:4538-4547. doi:10. 1002/pola.26266

23. Lamprecht A, Ubrich N, Hombreiro Perez M, Lehr CM, Hoffman M, Maincent P (2000) Influences of process parameters on nanoparticle preparation performed by a double emulsion ressure homogenization technique. Int J Pharm 196:177-182. doi:10.1016/S0378-5173(99)00422-6

24. Chandy T, Das GS, Wilson RF, Rao GHR (2002) Development of polylactide microspheres for protein encapsulation and delivery. J Appl Polym Sci 86:1285-1295. doi:10.1002/app.11139

25. Ishihara T, Takahashi M, Higaki M, Mizushima Y (2009) Efficient encapsulation of a water-soluble corticosteroid in biodegradable nanoparticles. Int J Pharm 365:200-205. doi:10.1016/j.ijpharm.2008. 08.030

26. Ma LQ, Hong XY, Liu ZG, Yuan WE (2012) Stabilisation and encapsulation of protein into biodegradable microspheres with zinc ion and protein in polyethylene glycol solution formed nanoparticles by freeze-drying. Micro Nano Lett 7:215-218. doi:10.1049/mnl.2011.0640

27. Ross AC, Taylor CL, Yaktine AL, Del Valle HB (2011) Dietary reference intakes for calcium and vitamin D. Committee to review dietary reference intakes for vitamin D and calcium. Institute of Medicine, National Academies Press, Washington DC 